\title{
Treatment of AIDS and HIV-Related Conditions-1999
}

\author{
Ronald H. Goldschmidt, MD, and Betty J. Dong, PharmD
}

During the last 2 years human immunodeficiency virus (HIV) disease and acquired immunodeficiency syndrome (AIDS) have been transformed from progressive, fatal diseases to ones in which the disease process can be improved. The striking effectiveness of combination antiretroviral therapy, especially regimens that include the protease inhibitor class of drugs, is largely responsible for this dramatic turnabout. Although some HIV-infected patients do not respond favorably to these therapies, and resistance to combination therapy can occur with time, there is now hope that if antiretroviral therapies continue to improve, HIV disease might eventually become another treatable chronic disease.

This Current Report - HIV updates our annual treatment guidelines. ${ }^{1}$ It is based on a review of the medical literature, our experience at San Francisco General Hospital, and experience gained from answering calls to our National HIV Telephone Consultation Service (Warmline). These guidelines are intended to provide treatment recommendations for antiretroviral therapy and associated medical problems of adults and adolescents with HIV disease and AIDS.

\section{Antiretroviral Therapy}

Guidelines for antiretroviral therapy are readily available. The United States Public Health Service publishes guidelines ${ }^{2}$ that are updated continually at http://www.cdc.gov or http://hivatis.org. Recommendations from the International AIDS Soci-

Submitted, revised, 3 September 1998.

From the Family Practice Residency Program, San Francisco General Hospital (RHG, BJD), and the Departments of Family and Community Medicine (RHG, BJD) and Clinical Pharmacy (BJD), University of California, San Francisco. Address reprint requests to Ronald $H$. Goldschmidt, $M D$, Family Practice Inpatient Service, San Francisco General Hospital, 1001 Potrero Ave, San Francisco, CA 94110. This article is also available at http://itsa.ucsf.edu/warmline.

Supported in part by the Pacific AIDS Education and Training Center, Grant No. 2 U69 PE00118-05, with the Bureau of Health Professions, Health Resources and Services Administration, Department of Health and Human Services. ety, ${ }^{3}$ the British HIV Association, ${ }^{4}$ and related articles add to the range of approaches to antiretroviral therapy. ${ }^{5-8}$

\section{Monitoring HIV Progression and Antiretroviral Therapy}

Refinements in viral load testing have revolutionized HIV care. The ultrasensitive assays of HIV RNA (viral load) levels can detect fewer than 50 or even 20 copies $/ \mathrm{mL}$, allowing the clinician and $\mathrm{pa}-$ tient to estimate activity of HIV disease and the degree of effectiveness of antiretroviral therapy. Although suppression of the viral load to less than the detectable level of the assay is the optimal goal of therapy, it is not always possible. Viral loads of less than about 500 copies $/ \mathrm{mL}$ are desirable. Decreases in viral load generally occur within 1 month after therapy begins, but maximal suppression can take up to 4 to 6 months of therapy. Antiretroviral therapy that does not suppress the viral load by at least $1 \log$ (10-fold) should be considered ineffective.

The CD4+ lymphocyte count remains an important surrogate marker in assessing HIV disease. Opportunistic infections rarely occur in patients with a CD4+ cell count of more than $200 / \mu \mathrm{L}$, and the conditions associated with severe disease generally occur when the $\mathrm{CD} 4+$ cell count is less than 50 to $100 / \mu \mathrm{L}$. The $\mathrm{CD} 4+$ count usually rises with effective antiretroviral therapy.

\section{Antiretroviral Drugs}

The three principal classes of antiretroviral drugs (Table 1) are the nucleoside reverse transcriptase inhibitors (NRTIs), the protease inhibitors, and the nonnucleoside reverse transcriptase inhibitors (NNRTIs). Combination antiretroviral therapy is usually based upon a foundation of two NRTIs plus protease inhibitor therapy. Monotherapy is never recommended because drug resistance will develop rapidly.

The most common combinations of NRTIs are zidovudine plus lamivudine, didanosine, or 
zalcitabine; and stavudine plus lamivudine or didanosine. Zidovudine and stavudine should not be used together because antagonism can occur. $\mathrm{Di}$ danosine and zalcitabine should not be used together because of additive toxicity.

The protease inhibitor class of drugs ${ }^{9}$ is most potent in decreasing viral load. When used in the absence of effective NRTI therapy, however, resistance usually occurs, rendering the protease inhibitors ineffective. Cross-resistance among protease inhibitors is common; when resistance develops to one protease inhibitor, resistance to other protease inhibitors can occur. Cross-resistance between ritonavir and indinavir is nearly 100 percent. Combination ritonavir plus saquinavir therapy can offer some benefits in persons who have protease inhibitor resistance to one other protease inhibitor. Protease inhibitors are associated with substantial side effects and important drug interactions, as listed in Table 1.

The NNRTIs can also be potent inhibitors of HIV replication. Without concomitant and effective NRTI therapy, however, the virus will rapidly develop complete resistance to NNRTI therapy. NNRTI resistance occurs across this class of drugs, so that resistance to one NNRTI results in resistance to the others. Currently, the most common role of the NNRTIs is to substitute for a protease inhibitor when toxicity or resistance to protease inhibitor therapy has occurred, but some providers use a combination of NRTIs plus protease inhibitor plus NNRTI as initial therapy or even NRTIs plus NNRTI as initial therapy. Longterm studies are needed to evaluate these strategies.

Hydroxyurea ${ }^{10}$ has been used in combination with didanosine with apparent benefit in both early HIV infection and in salvage therapy. Whether hydroxyurea has long-term benefits and whether it is effective in combination with other antiretroviral agents remains unknown. The role of the nucleotide analog adefovir is unclear at this time. Adefovir has been used with apparent effectiveness as an additional agent in salvage therapy in patients who have failed other regimens.

Pseudo-Cushing syndrome, which includes the development of "buffalo hump," abnormal fat deposition, systemic dyslipidemias, and glucose intolerance and insulin resistance, has been described as a side effect of effective antiretroviral therapy. ${ }^{11,12}$ These complications occur more commonly with combination therapy that includes protease in- hibitors than with other combination regimens, but the syndrome also can occur in the absence of protease inhibitors. The pathophysiology of these side effects of therapy is not understood, although various hypotheses have been suggested. ${ }^{11,13}$ It is possible that the renourishment of patients that results from effective combination antiretroviral therapy might be a cause of this syndrome. ${ }^{11}$ These abnormalities are important for both cosmetic and possible long-term cardiovascular reasons.

\section{Initiating Antiretroviral Therapy}

The optimal time to initiate antiretroviral therapy remains controversial. ${ }^{2-4}$ The prevailing philosophy in the United States is to begin highly potent combination (three-drug) antiretroviral therapy as early as possible for patients with detectable virus who are prepared to commit to a lifetime of antiretroviral therapy. Reducing viral burden results in fewer infected cells, less immune system destruction, and decreased disease progression. By maximally suppressing viral replication, it is hoped that resistance to antiretroviral drugs will also be minimized. The Public Health Service recommends beginning therapy for patients with a CD4+ cell count of fewer than $500 / \mu \mathrm{L}$ or HIV RNA levels of greater than 10,000 copies/mL (bDNA assay) or 20,000 copies/mL (RT-PCR assay). ${ }^{2}$ The International AIDS Society uses a threshold of 5000 to 10,000 copies $/ \mathrm{mL}$. ${ }^{3}$ The British HIV Association Guidelines emphasize that therapy should be started before irreversible damage of the immune system has occurred, specifically at a CD4+ cell count of more than $350 / \mathrm{LL}^{4}$

\section{Adherence}

Close adherence to antiretroviral drug regimens is notoriously difficult, yet critical to treatment success. Interruption of therapy can result not only in ineffective therapy but in the rapid induction of drug resistance. Failure of early treatment can result in the emergence of a virus that is resistant not only to the drugs being taken but to that entire class of drugs. Second- or third-choice (salvage) regimens are generally less effective than initial regimens. The patient who is not prepared or cannot adhere to lifelong complicated medical regimens could be harmed, rather than helped, by initiation of combination antiretroviral therapy, ${ }^{14}$ a fact that should weigh heavily on primary clinicians when recommending antiretroviral therapy, 
especially for asymptomatic persons who have a low probability of developing AIDS within a few years. Although it is difficult for the clinician to withhold potentially beneficial therapy, in some cases this strategy can be the most prudent until the clinician and patient believe regimen adherence can be maintained.

\section{Changing Antiretroviral Regimens}

Antiretroviral regimens are considered to be failed regimens if patients develop progressive opportunistic conditions of advancing AIDS, when the viral load increases substantially (at least $0.5 \mathrm{log}$ ), or when the CD4+ count falls. When the viral load has been undetectable, development of new detectable virus in the serum is also considered to be a sign of failed therapy. Long-term studies will be needed to determine the proper time to change therapy, as clinical improvement sometimes can be sustained beyond the time when surrogate markers (viral load and CD4+ count) deteriorate.

When changing antiretroviral drugs, at least two drugs should be changed. Optimally, an entirely new regimen with at least three new drugs is used. ${ }^{2-4}$ When a protease inhibitor-containing regimen has failed, changing from that protease inhibitor to NNRTI therapy or possibly to combination protease inhibitor therapy (in addition to changing two NRTIs) is needed. Changing between ritonavir and indinavir and between the NNRTIs should be avoided because of crossresistance.

\section{Effects of Antiretroviral Therapy on AIDS-Related Conditions}

Effective antiretroviral therapy can have profound effects on AIDS-related conditions as well as the progression of HIV disease. Both the incidence and the natural history of such conditions as Pneumocystis carinii pneumonia, Kaposi sarcoma, cytomegalovirus disease, progressive multifocal leukoencephalopathy, mycobacterial disease, and AIDS dementia have been altered favorably by antiretroviral therapy. ${ }^{15}$ With reconstitution of elements of the immune system, however, there are also reports of flare-ups of underlying disease ${ }^{16}$ as the improved immune system stimulates a more vigorous response against these infections than had occurred during the more immune-depleted state. Although these flare-ups, such as retinitis and inflammatory responses to indolent mycobacterial disease, are usually temporary, clinicians will need to be alert to these unexpected manifestations.

Prophylaxis against opportunistic infections ${ }^{17}$ remains a mainstay in HIV primary care. Most important is primary prophylaxis against $P$ carinii pneumonia, which is recommended for all HIVinfected persons with symptomatic HIV disease or AIDS, including those with CD4+ cell counts of less than 200/ $\mathrm{LL}$. Prophylaxis against Mycobacterium avium complex (MAC) disease has been recommended after CD4+ cell counts decrease to less than $50 / \mu \mathrm{L}$. Primary prophylaxis against candidal and other fungal diseases, herpes infections, and cytomegalovirus disease is not routinely recommended.

CD4+ count thresholds for opportunistic infection prophylaxis are based on the $\mathrm{CD} 4+$ count nadir. For patients whose $\mathrm{CD} 4+$ counts rise above those thresholds after initiation of antiretroviral therapy, prophylaxis against opportunistic infections usually should be continued. Studies are in progress to determine whether less intensive prophylaxis regimens might be adequate in persons receiving effective antiretroviral therapy. Some studies indicate these strategies can be effective. ${ }^{18,19}$ Effective antiretroviral therapy will ultimately result in changes in recommendations for both primary prophylaxis and maintenance therapy after treatment of acute infections, but until more definitive long-term studies show that prophylaxis regimens can be altered, current recommendations should remain in effect.

\section{Tuberculosis}

Tuberculosis prophylaxis is indicated for HIV-infected persons who have an induration of $5 \mathrm{~mm}$ or greater on the standard tuberculin skin test. Isoniazid prophylaxis does not require changing antiretroviral regimens, but the shorter rifampin- or rifabutin-based regimens require special drug-interaction considerations. In addition, treatment of active tuberculosis will result in potential drug interactions with antiretroviral medications. Consultation with a tuberculosis expert and adherence to new guidelines ${ }^{20}$ can avoid serious treatment problems.

\section{The Table}

Table 1 gives our recommendations for treating specific diseases and the major symptoms of HIV infection and AIDS. The recommendations are

Text contimued on page 93 


\begin{tabular}{lll} 
General/Systemic p. 74 & Ophthalmologic p. 83 & Gastrointestinal p. 86 \\
Skin/Mucocutaneous p. 81 & Oral Cavity p. 84 & Pulmonary p. 87 \\
Hematologic p. 83 & Esophageal p. 85 & Central Nervous System p. 90 \\
\hline
\end{tabular}

System, Problem, and Drug Regimen Duration

Adverse Effects/Drug Interactions

Comments

GENERAL/SYSTEMIC Antiretroviral (Anti-HIV)

Combination therapies

Combination therapy is always recommended, as monotherapy is ineffective and promotes drug resistance. Most common regimens include two nucleoside reverse transcriptase inbibitors (NRTIs) along with protease inbibitor (PI) therapy. Nonnucleoside reverse transcriptase inbibitor (NNRTI) therapy can substitute for protease inbibitor therapy for patients who cannot take or are resistant to protease inbibitor therapy, or can be added to NRTI plus PI regimens when necessary. Cross-resistance among PIs is common, as is cross-resistance among NNRTLs. NRTI combinations are: zidovudine plus lamivudine, didanosine, or zalcitabine; or stavudine plus lamivudine or didanosine. Zidorudine and stavudine should not be used in combination. PI therapy is usually with nelfinavir, indinavir, ritonavir, saquinavir soft-gel capsules, or ritonavir plus saquinavir. Indinavir and saquinavir should not be used in combination. See text for further discussion.

Nucleoside reverse transcriptase inbibitors (NRTLs)

Zidovudine (AZT, Retrovir) $200 \mathrm{mg}$ po tid or $300 \mathrm{mg}$ po bid; lower dosages (eg, $100 \mathrm{mg} 3$ times daily) for patients unable to tolerate higher dosages and patients with renal failure or cirrhosis. Available as liquid formulation. Available also as fixeddose combination (Combivir) consisting of zidovudine $(300 \mathrm{mg}$ ) with lamivudine $(150 \mathrm{mg}$ ) given as 1 capsule bid

Until efficacy

wanes or toxicity occurs 
GENERAL/SYSTEMIC

Antiretroviral (Anti-HIV) (cont.)

Zalcitabine (ddC, Hivid) $0.75 \mathrm{mg}$ po tid; $0.375 \mathrm{mg}$ po tid for patients $<30 \mathrm{~kg}$. Dosage reduction in renal failure

Stavudine (d4T, Zerit) 20 - $40 \mathrm{mg}$ po bid for patients > $60 \mathrm{~kg}$; $15-30 \mathrm{mg}$ po bid for patients $40-60 \mathrm{~kg}$ reduce dosage for patients $<40 \mathrm{~kg}$ and for patients with renal failure. Available as liquid formulation

Lamivudine (3TC, Epivir) $150 \mathrm{mg}$ po bid; $2 \mathrm{mg} / \mathrm{kg}$ po bid for patients $<50 \mathrm{~kg}$ Dosage reduction in renal failure. Available as liquid formulation. Available also as fixed dose combination (Combivir) consisting of zidovudine $(300 \mathrm{mg}$ ) with lamivudine $(150 \mathrm{mg})$ given as 1 capsule bid

\section{Until efficacy} wanes or toxicity occurs

Painful peripheral neuropathy (dosage related, reversible); rash; stomatitis, aphthous ulcers; pancreatitis; esophageal ulceration; seizures; aminotransferase elevations; cardiomyopathy

\section{Drug interactions}

Avoid alcohol and other pancreatic toxins (eg, systemic pentamidine). Avoid concomitant neurotoxic drugs (eg, didanosine, stavudine, isoniazid, vinca alkaloids, oral ganciclovir)

Until efficacy wanes or toxicity occurs

Painful peripheral neuropathy; aminotransferase elevations; anemia, macrocytosis; psychological disturbances, insomnia, anxiety, panic attacks

\section{Drug interactions}

Avoid concomitant use of drugs that can cause neurotoxicity or pancreatic toxicity. See didanosine

Until efficacy wanes or

Headache, fatigue, insomnia; peripheral neuropathy, muscle aches; toxicity occurs rash; aphthous ulcers; nausea; rare neutropenia, thrombocytopenia; paronychia
Zalcitabine might be less potent than other NRTIs

Dosage range in this table is lower than standard dosage ( $40 \mathrm{mg}$ po bid), as studies suggest these lower dosages are associated with equivalent efficacy and a lower incidence of peripheral neuropathy

Do not use in combination with zidovudine

Provides some efficacy against hepatitis B. Once-daily dosing ( $300 \mathrm{mg}$ po qd) under investigation
Abacavir (1592U89, Ziagen) $300 \mathrm{mg}$ po bid with or without food
Until efficacy wanes or toxicity occurs
Nausea, headache, malaise; abdominal pain, diarrhea, rash. Hypersensitivity reaction $(2 \%-5 \%$, usually in first 4 weeks): flu-like symptoms, fever, malaise, abdominal cramping, nausea, vomiting, diarrhea, morbilliform rash, elevations in transaminases and creatine kinase levels. Symptoms resolve if drug stopped. Do not rechallenge, as anaphylactic reactions and deaths reported

\section{Protease inbibitors (PIs)}

Nelfinavir (Viracept) $750 \mathrm{mg}$ po tid with meals or $1250 \mathrm{mg}$ po bid with meals. Available as liquid formulation
Until efficacy wanes or toxicity occurs
Diarrhea; hypertriglyceridemia; hypercholesterolemia, abnormal fat accumulation, hyperglycemia

Drug interactions

P-450 enzyme inhibitor. Avoid concomitant use with rifampin, rifabutin (or decrease rifaburin dosage to $150 \mathrm{mg}$ po qd), astemizole, terfenadine, and cisapride. Benzodiazepine interactions under investigation. Avoid simvastatin or lovastatin because of rhabdomyolysis; can use pravastatin or fluvastatin
Resistance develops slowly; resistant strains might be sensitive to other PIs 
System, Problem, and Drug Regimen

Duration

Adverse Effects/Drug Interactions

Comments

GENERAI/SYSTEMIC

Antiretroviral (Anti-HIV) (cont.)

Indinavir (Crixivan)

$800 \mathrm{mg}$ po $\mathrm{q} 8 \mathrm{~h}$ on

tea, toast; dosage

adjustment to $600 \mathrm{mg}$

po $q \mathrm{~h}$ in hepatic

disease empty stomach or with

skim milk, juice, coffee,

\section{Until efficacy Nephrolithiasis, crystalluria, inter- wanes or toxicity occurs stitial nephritis; hypertriglyceridemia, hypercholesterolemia, abnormal fat accumulation, hyperglycemia; nausea, vomiting, diarrhea, abdominal pain; asymptomatic hyperbilinubinemia, aminotransferase elevations; rash; insomnia, headache, dizziness, taste disturbances; thrombocytopenia}

\section{Drug interactions}

Avoid concomitant use of indinavir with rifampin, rifabutin (or decrease rifabutin dosage to $150 \mathrm{mg}$ po qod), astemizole (Hismanal), cisapride (Propulsid), triazolam (Halcion), or midazolam (Versed). Decrease indinavir dosage to $600 \mathrm{mg}$ po $\mathrm{q} 8 \mathrm{~h}$ when given with ketoconazole or delavirdine. Indinavir administration must be at least 1 hour apart from didanosine or antacid administration. Avoid simvastatin or lovastatin because of rhabdomyolysis; can use pravastatin or fluvastatin

Ritonavir (Norvir) $600 \mathrm{mg}$ po bid with meals; can increase from $300 \mathrm{mg}$ po bid to $600 \mathrm{mg}$ po bid over 4 - 7 days to minimize gastrointestinal symptoms. Available as liquid formulation. Combination PI therapy with saquinavir permits lower dosages of each (see below)

Saquinavir soft-gel capsules (Fortovase) 1200 mg po tid with meals Combination PI therapy with ritonavir permits lower dosages of each (see below)

Until efficacy Nausea, vomiting, diarrhea, anorexia wanes or toxicity occurs in more than $50 \%$ of patients; aminotransferase elevations; hypertriglyceridemia, hypercholesterolemia, abnormal fat accumulation, hyperglycemia; fatigue, weakness, headache, dizziness, circumoral paresthesias; hyperuricemia, increased creatine kinase; taste disturbances

\section{Drug interactions}

Potent hepatic $\mathrm{P}-450$ enzyme inhibitor. Avoid concomitant use with rifampin, rifabutin (or decrease rifabutin dosage to $150 \mathrm{mg}$ po qod), astemizole, cisapride, and benzodiazepines except lorazepam and temazepam. Dosages of desipramine and other antidepressants, narcotics, and oral contraceptives might need adjustment. Avoid simvastatin or lovastatin because of rhabdomyolysis; can use pravastatin or fluvastatin
Until efficacy wanes or toxicity occurs

Headache, confusion; hypertriglyceridemia, hypercholesterolemia, abnormal fat accumulation, hyperglycemia; nausea diarrhea, abdominal pain; fever

Drug interactions

Ketoconazole, ritonavir, delavirdine, and grapefruit juice increase saquinavir serum concentration. Avoid concomitant use of saquinavir with indinavir, rifampin, rifabutin, phenytoin, carbamazepine, phenobarbital, efavirenz, dexamethasone, nevirapine, and other enzyme inducers. Avoid simvastatin or lovastatin because of rhabdomyolysis; can use pravastatin or fluvastatin
Take with at least 6 glasses of noncaffeinated liquid daily to avoid nephrolithiasis

Must be taken every 8 hours, not 3 times daily

Capsules must be refrigerated; solution is stable at room temperature for 30 days

Hard-gel formulation (Invirase, $600 \mathrm{mg}$ po tid within 2 hours of a high-fat meal to increase absorption); not recomended because of poor bioavailability $(4 \%)$, even when taken with high-fat meal 
GENERAL/SYSTEMIC

Antiretroviral (Anti-HIV) (cont.)

Ritonavir $400-600 \mathrm{mg}$ po bid

plus
Until efficacy See individual agents
wanes or

toxicity occurs
Saquinavir soft-gel capsules $400-600 \mathrm{mg}$ po bid. Lower dosages (400 $\mathrm{mg}$ of each) preferred

$\begin{array}{ll}\text { Nelfinavir } 750 \mathrm{mg} \text { po tid } & \begin{array}{l}\text { Until efficacy See individual agents } \\ \text { wanes or } \\ \text { toxicity occurs }\end{array} \\ \text { plus } & \end{array}$

Saquinavir $800 \mathrm{mg}$ po tid

Amprenavir (141W94, Agenerase) $1200 \mathrm{mg}$ po bid with or without food

Until efficacy wanes or toxicity occurs
Nonnucleoside reverse transcriptase inbibitors (NNRTIs)

Nevirapine (Viramune) $200 \mathrm{mg}$ po qd for 14 days; if no rash develops, increase to $200 \mathrm{mg}$ po bid. Once-daily dosing (400 mg po qd) under investigation.

Delavirdine (Rescriptor) $400 \mathrm{mg}$ po tid. Can dissolve in $3 \mathrm{oz}$ water as slurry

Efavirenz (DMP 266, Sustiva) $600 \mathrm{mg}$ po ghs with or without food; $200 \mathrm{mg}$ po tid if dizziness occurs
Until efficacy Maculopapular rash, Stevens-Johnson syndrome; nausea, vomiting, diarrhea; toxicity occurs fatigue, fever, headaches; aminotransferase elevations; rare hematologic toxicity

\section{Drug interactions}

$\mathrm{P}-450$ enzyme inducer, avoid concomitant use with saquinavir, rifampin, and rifabutin

Until efficacy wanes or toxicity occurs

Maculopapular rash; nausea; headache; aminotransferase elevations especially when taken with saquinavir; neutropenia when taken with nelfinavir

\section{Drug interactions}

P-450 enzyme inhibitor. Avoid concomitant use of astemizole, rifampin, rifabutin, phenytoin, carbamazepine, cisapride, alprazolam, midazolam, triazolam, ergot alkaloids. Ketoconazole, itraconazole, fluconazole, clarithromycin, and fluoxetine can increase delavirdine serum concentrations; dosage reduction might be necessary

\section{Until efficacy Dizziness, anxiety, inability to} wanes or concentrate, lightheadedness, headache toxicity occurs dysphoria, nightmares; nausea; rash (less than other NNRTIs). Avoid in pregnancy

Drug interactions

Avoid use with saquinavir, astemizole, and cisapride. Increased indinavir dosage to $1 \mathrm{~g}$ po $\mathrm{q} 8 \mathrm{~h}$ might be required

\section{Generally well tolerated}

Combination therapy provides higher saquinavir levels

\section{Generally well tolerated}

Combination therapy provides higher saquinavir levels

\section{Approval expected during 1999}

Discontinue drug at any time if rash is severe. Do not increase dosage if any rash is present during first 14-day lead-in period

Rash from one NNRTI does not predict rash from other NNRTIs

Delavirdine increases saquinavir and indinavir levels by $50 \%$. Reduce indinavir dosage to $600 \mathrm{mg}$ po $\mathrm{q} 8 \mathrm{~h}$ when used in combination with delavirdine. Separate didanosine or antacid administration from delavirdine administration by at least 1 hour

Rash from one NNRTI does not predict rash from other NNRTIs

Good central nervous system penetration; resistance might develop more slowly than other NNRTIs.

Rash from one NNRTI does not predict rash from other NNRTIs

Continued 
Table 1. Continued

System, Problem, and

Drug Regimen

Duration

Adverse Effects/Drug Interactions

Comments

GENERAL/SYSTEMIC

Antiretroviral (Anti-HIV) (cont.)

Otber Agents

Hydroxyurea (Hydrea) $500 \mathrm{mg}$ po bid

Until efficacy

wanes or

Bone marrow suppression including toxicity occurs hydroxyurea therapy

\section{Adefovir dipivoxil (bis- POM PMEA, Preveon) $120 \mathrm{mg}$ po qd; lower dosage ( $60 \mathrm{mg}$ po $\mathrm{qd}$ ) might be effective}

Postexposure prophylaxis

Zidovudine $200 \mathrm{mg}$ po tid plus lamivudine $150 \mathrm{mg}$ po bid with or without nelfinavir $750 \mathrm{mg}$ po tid or indinavir $800 \mathrm{mg}$ po $q 8 \mathrm{~h}$

\section{Pregnancy}

Zidovudine-containing antiretroviral regimen during pregnancy, plus intrapartum zidovudine $2 \mathrm{mg} / \mathrm{kg}$ IV for 1 hour, then $1 \mathrm{mg} / \mathrm{kg} / \mathrm{h}$ until delivery

\section{Wasting Syndrome}

Megestrol (Megace) suspension $(40 \mathrm{mg} / \mathrm{mL})$ $800 \mathrm{mg}$ po qd

Dronabinol (Tetrahydrocannabinol [THC],

Marinol) $2.5 \mathrm{mg}$ po bid 30 minutes to 1 hour before meals. Maximum $20 \mathrm{mg}$ qd

Human growth hormone (r-hGH, Serostim)

$0.1 \mathrm{mg} / \mathrm{kg} / \mathrm{d} \mathrm{SQ}$

(average dosage $6 \mathrm{mg} / \mathrm{d}$ )

Anabolic steroids (eg, testosterone $200 \mathrm{mg} \mathrm{IM}$

every 2 weeks or $300 \mathrm{mg}$

IM every 3 weeks, oxandrolone [Oxandrin]

$2.5 \mathrm{mg}$ po bid - tid or

testosterone patches

[Testoderm, Androderm])

Nausea, vomiting, diarrhea, aminotransferase elevations; malaise; renal insufficiency, Fanconi-like syndrome with hypophosphatemia, glycosuria, renal acidosis, proteinuria, and elevated serum creatinine

\section{Drug interactions}

Avoid concurrent use with other nephrotoxins

\section{4 weeks See above adverse effects and drug} interactions. Zidovudine and lamivudine appear to be safe in pregnancy
Until end of pregnancy

Indefinitely

Indefinitely

Restlessness, irritability, insomnia, dizziness, loss of coordination, psychotomimetic effects; fatigue; tachycardia

Unknown

Arthralgias, joint stiffness, carpal tunnel syndrome; hyperglycemia; hypertriglyceridemia

Unknown

Edema; cholestatic jaundice, peliosis hepatis, aminotransferase elevations; increased libido, testicular atrophy, priapism; insomnia
Can be used in combination with didanosine (and possibly other antiretroviral drugs) as salvage therapy. Long-term risks and benefits unknown

Nucleotide analog. Role unclear at this time; might offer benefit in salvage therapy. Carnitine supplementation might be required. Approval expected during 1998. Available through expanded access at $1-800-445-3235$

Administration within $1-2$ hours or as soon as possible after exposure. Can substitute other antiretroviral agents (eg, stavudine plus didanosine) when source patient has received extensive treatment with zidovudine or lamivudine. Add nelfinavir, indinavir, or other PI for high-risk exposures and when source patient suspected to have developed antiretroviral drug resistance. Can call 1-888-HIV-4911 for additional assistance

Zidovudine therapy, combined with intrapartum intravenous zidovudine plus oral zidovudine $2 \mathrm{mg} / \mathrm{kg}$ qid to infants for first 6 weeks of life, decreases transmission to infants

Unconfirmed reports of premature deliveries when PI therapy is used

Megestrol can increase appetite and cause fat accumulation with weight gain. Uncertain whether this weight gain improves health. Available also as tablets, but large number of tablets required for administration and more expensive

Increases appetite and can cause weight gain. Uncertain whether this weight gain improves health. Antinauseant. Not recommended for persons sensitive to marijuana effects

Can improve exercise endurance and increase weight, characterized by increased lean body mass and decreased fat

Might improve well-being and increase lean body mass. Treatment should be accompanied by exercise 
Table 1. Continued

System, Problem, and

Drug Regimen

Duration

Adverse Effects/Drug Interactions

Comments

GENERAL/SYSTEMIC

Mycobacterium avium

complex (MAC)

Propbylaxis

Clarithromycin (Biaxin) Indefinitely

$500 \mathrm{mg}$ po bid

OR

Azithromycin

(Zithromax) $1200 \mathrm{mg}$

po once weekly or

$500 \mathrm{mg}$ po qd

OR

Rifabutin (Mycobutin) $300 \mathrm{mg}$ po qd

Acute MAC disease

Ethambutol (Myambutol)

$15 \mathrm{mg} / \mathrm{kg}$ po $\mathrm{qd}(1 \mathrm{~g}$

po qd maximum); dosage

reduction in renal failure (minimum of 12 weeks)

plus either

Clarithromycin $500 \mathrm{mg}$ po

bid. Higher dosages

associated with higher

mortality

or

Azithromycin $500 \mathrm{mg}$ po qd

For serious illness or failure to

respond within I month, can

add one or two of the following:

Rifabutin $300 \mathrm{mg}$ po qd Indefinitely
Indefinitely, if tolerated

Clarithromycin and azithromycin side effects include nausea, vomiting, dyspepsia, diarrhea, hearing loss, aminotransferase elevations

Indefinitely

Drug interactions

Clarithromycin increases serum levels of rifabutin and can lead to rifabutin toxicity, including severe anterior uveitis. Clarithromycin and azithromycin increase levels of carbamazepine and theophylline. Avoid astemizole in combination with azole antibiotics because of increased risk of torsades de pointes and ventricular tachyarrhythmias

Nausea (can be reduced by administering $150 \mathrm{mg}$ po bid). Rash. Uveitis with dosages > $300 \mathrm{mg}$ po qd and in patients receiving concomitant clarithromycin, fluconazole, or indinavir therapy. Red-orange discoloration of body fluids. Rare neutropenia, thrombocytopenia, anemia; flu-like syndrome; elevated bilirubin and alkaline phosphatase levels, hepatitis

Drug interactions

Multiple interactions with protease inhibitors (see Antiretroviral drugs, above). Rifabutin increases metabolism of methadone, zidovudine, and clarithromycin; higher dosage of these drugs might be required. Clarithromycin increases rifabutin blood levels and can lead to rifabutin toxicity

Optic neuritis (if > $25 \mathrm{mg} / \mathrm{kg} / \mathrm{d}$ ); hyperuricemia; nausea, vomiting
Survival benefits shown for clarithromycin. Prophylaxis can be offered to patients with CD $4+$ cell counts $<50 / \mu \mathrm{L}$

Clarithromycin and rifabutin might pro vide prophylaxis against Cryptosporidium

Exclude Mycobacterium tuberculosis infection before initiating rifabutin therapy

Treatment indicated for documented MAC disease and patients with progressive signs, symptoms, and laboratory abnormalities consistent with MAC disease. Clinical improvement might take 2 - 4 weeks. Isolation of MAC in stool or sputum might not indicate systemic disease but is usually treated with ethambutol plus a macrolide antibiotic

When both $M$ tuberculosis and MAC infections are suspected, add isoniazid, rifampin, and pyrazinamide to ethambutol and clarithromycin pending culture results. See $M$ tuberculosis

Rifampin (Rimactane, Rifadin) 450 - 600 $\mathrm{mg}$ po qd can be substituted for rifabutin if concerned about $M$ tuberculosis

Continued 
System, Problem, and

\section{GENERAL/SYSTEMIC \\ Mycobacterium avium complex (MAC) (cont.)}

Ciprofloxacin Indefinitely

(Cipro) $500-750 \mathrm{mg}$

po qd - bid
Nausea, vomiting, diarrhea. Reversible pink to brown-black discoloration of skin, eyes, body secretions; rash. Hyperglycemia. Retinal degeneration

\section{Drug interactions}

Binds to cations, resulting in decreased ciprofloxacin absorption. Administer 2- 4 hours after antacids, sucralfate, dairy products, and didanosine

\section{Amikacin (Amikin) 7.5 - $\quad 2$ - 8 weeks \\ Nephrotoxicity, ototoxicity} $10.0 \mathrm{mg} / \mathrm{kg} \mathrm{IM} / \mathrm{TV}$ qd

\section{Mycobacterium tuberculosis}

\section{Propbylaxis}

Isoniazid (NH)

$300 \mathrm{mg}$ po qd

$6-12$ months

OR

Rifampin or rifabutin
plus
Pyrazinamide or
isoniazid
Active tuberculasis
Combinations of
isoniazid, rifampin or
rifabutin, pyrazinamide,
ethambutol, and
streptomycin

$2-3$ months

Begin with 4

drugs. After 2

months can

usually continue

2-drug therapy, depending upon susceptibility

testing results.

Total treatment: at least 6 months, and 6 months beyond culture conversion

\section{Histoplasmosis}

Acute

Amphotericin B

(Fungizone) $1.0 \mathrm{mg} /$

$\mathrm{kg}$ IV qd until $15 \mathrm{mg}$ '

$\mathrm{kg}$ total dosage has been administered. Decrease to $0.7-0.8 \mathrm{mg} / \mathrm{kg}$ qd if not tolerated

followed by

Itraconazole (Sporanox) See above $200 \mathrm{mg}$ po bid

\section{6 - 8 weeks total See CENTRAL NERVOUS SYSTEM, acute therapy \\ Cryptococcus neoformans} (amphotericin plus itraconazole) possibly other antiretroviral drugs
Monitor drug levels in patients with renal failure

Prophylaxis for all HIV-infected persons with $\geq 5$-mm intermediate-strength tuberculin skin test induration and those with strong history of tuberculosis exposure regardless of skin test reactivity

\section{Drug interactions}

Increases metabolism of ketoconazole; larger dosages of ketoconazole might be required. Increased phenytoin and carbamazepine toxicity; monitor levels

See individual drug toxicities

Drug interactions

PIs should not be administered concurrently with rifampin. Rifabutin contraindicated with ritonavir, soft-gel saquinavir, delavirdine, and possibly other antiretroviral drugs

\section{See individual drug adverse effects} and drug interactions

\section{Drug interactions}

PIs should not be administered concurrently with rifampin. Rifabutin contraindicated with ritonavir, soft-gel saquinavir, delavirdine, and

Isoniazid can be administered concurrently with NRTIs, PIs, NNRTIs

When short-course prophylaxis is administered with or without directly observed therapy (DOT), consultation with tuberculosis experts is recommended. Effective antiretroviral therapy should not be discontinued to permit use of specific antituberculosis drugs

Consultation with tuberculosis experts required. Treatment guidelines available through Centers for Disease Control and Prevention at http://www.cdc.gov

Amphotericin B recommended initially; oral therapy does not appear as effective. Itraconazole $200 \mathrm{mg}$ po bid might be effective

Nausea, vomiting; hypokalemia; hyper- Teratogenic tension; aminotransferase elevations; adrenal insufficiency; rhabdomyolysis. 
System, Problem, and Drug Regimen

\section{GENERAL/SYSTEMIC}

Histoplasmosis (cont.)

Itraconazole (cont.)

\author{
Maintenance \\ Itraconazole $200 \mathrm{mg}$ po qd Indefinitely \\ OR \\ Amphotericin B $50 \mathrm{mg}$ IV Indefinitely \\ each week, 2 times a week, \\ or every other week \\ Coccidioidomycosis \\ Acute \\ Amphotericin B (as above) 6-8 weeks \\ or \\ Indefinitely \\ Fluconazole \\ Indefinitely \\ Fluconazole
}

\section{Cryptococcosis}

\section{SKIN/MUCOCUTANEOUS}

\section{Kaposi sarcoma}

Observation

OR

Local treatment (radiation therapy, cryotherapy, excision, or intralesional vinblastine)

\section{OR}

Systemic chemotherapy with vinblastine and vincristine, vincristine alone, or combination of doxorubicin, bleomycin, and vincristine

OR

Interferon-alpha $3 \mathrm{mU}$ SQ 3 times weekly; increase by $3 \mathrm{mU} / \mathrm{d}$ every 2 weeks as tolerated (maximum $27 \mathrm{mU} / \mathrm{d}$ )

\section{Seborrheic dermatitis}

Hydrocortisone (HC) cream $2.5 \%$ plus

ketoconazole cream

$2 \%$ bid; severe cases

can require ketoconazole

$200-400 \mathrm{mg}$ po qd for

3 - 4 weeks

\author{
Drug interactions \\ Potent hepatic enzyme inducers, such \\ as rifampin and phenytoin, increase \\ metabolism of itraconazole; higher \\ itraconazole dosages might be required. \\ Avoid concurrent use with triazolam, \\ alprazolam (Xanax), antacids, $\mathrm{H}_{2}$ \\ blockers, and omeprazole
}

Fluconazole $400 \mathrm{mg}$ po qd less effective

Optimum frequency of administration not determined

See CENTRAL NERVOUS SYSTEM Fluconazole penetrates CNS and is Cryptococcus neoformans preferred initial therapy for CNS coccidioidomycosis
Indefinitely

\section{See CENTRAL NERVOUS SYSTEM, Cryptococcus neoformans}

\section{Mucositis in head and neck regions} from radiation therapy

Until lesions and symptoms are resolved or controlled

Same

Usual chemotherapeutic agent side effects. Liposomal preparations might have some advantages in specific cases

Indefinitely

Fatigue, myalgia, asthenia; neutropenia, thrombocytopenia; aminotransferase elevations

Until resolved See ORAL CAVTTY, Candida albicans, ketoconazole
Treatment not required unless lesions are symptomatic or cosmetically bothersome

Treatment effective for cosmetic purposes, for relief of symptoms, and to help reduce edema caused by lymphatic obstruction

Therapy can help control disease but does not alter prognosis. Consultation by oncologist or AIDS specialist usually required

Hematologic toxicities increased in parients taking zidovudine. Dosages greater than $10 \mathrm{mU} / \mathrm{d}$ necessary for efficacy

Commonly involves face, eyebrows, retroauricular areas, nasolabial folds, and scalp. Addition of antifungal cream enhances therapeutic response and reduces the frequency of steroid application 
System, Problem, and Drug Regimen

\section{SKIN/MUCOCUTANEOUS (cont.) \\ Mucocutaneous herpes simplex}

Acute

$$
\begin{aligned}
& \text { Acyclovir (Zovirax) } 7-10 \text { days } \\
& 200 \mathrm{mg} \text { po } 5 \text { times a day } \\
& \text { or } 400 \mathrm{mg} \text { po tid }
\end{aligned}
$$

OR

Valacyclovir (Valtrex) 10 days

$500 \mathrm{mg}-1 \mathrm{~g}$ po bid

OR

Famciclovir (Famvir) $250 \mathrm{mg}$ po tid

\section{Maintenance}

Acyclovir $200-400 \mathrm{mg}$ po Indefinitely

$2-3$ times a day or

valacyclovir $500 \mathrm{mg}$ po bid

or $1 \mathrm{~g}$ po qd or famciclovir

$250 \mathrm{mg}$ po bid

Disseminated, extensive, or persistent herpes simplex

Acyclovir $5 \mathrm{mg} / \mathrm{kg} /$ dose IV q 8 h; dosage 7 - 14 days or reduction in renal failure; resolve maintenance as above

OR

Valacyclovir $1 \mathrm{~g}$ po tid

7 - 14 days or until lesions resolve

Herpes zoster

(shingles, disseminated, or persistent zoster)

Acyclovir $10 \mathrm{mg} / \mathrm{kg} /$ dose IV q $8 \mathrm{~h}$; or acyclovir 800 mg po 5 times a day; reduce dosage of intravenous acyclovir in renal failure

$7-10$ days or until lesions

resolve

OR

Valacyclovir $1 \mathrm{~g}$ po tid

7 - 10 days

\section{Acyclovir-resistant} herpes infections

Foscarnet $40 \mathrm{mg} / \mathrm{kg} /$ dose IV q $8 \mathrm{~h}$; dosage reduction in renal failure

10 - 14 days or until lesions clear

OR

Trifluridine (Viroptic) Same $1 \%$ solution $\mathrm{q} 8 \mathrm{~h}$

Cidofovir

Same
Oral: nausea, vomiting, diarrhea, dizziness

Nausea, vomiting, diarrhea; headache, dizziness, fatigue, insomnia. Hemolytic uremic syndrome (if $>3 \mathrm{~g} / \mathrm{d}$ )

Nausea, vomiting, diarrhea; headache, dizziness, fatigue, insomnia

Intravenous: lethargy, tremors, confusion, hallucinations; phlebitis; increased serum creatinine, reversible crystalline nephropathy

\section{See above}

See OPHTHALMOLOGIC, CMV below

\section{See OPHTHALMOLOGIC, CMV,} below

Severe herpes infections (eg, esophagitis, colitis, encephalitis) require intravenous acyclovir. Maintain good urine output and hydration to prevent acyclovir crystallization

Alternate drugs are foscarnet, vidarabine, cidofovir, and trifluridine (Viroptic) applied to skin covered with polymyxin B-bacitracin (Polysporin) ointment q $8 \mathrm{~h}$. Keratoconjunctivitis requires more frequent $(q 2 h)$ trifluridine application

Rare hypersensitivity reactions

See OPHTHALMOLOGIC, CMV, below
Apply to affected areas and cover with antibiotic ointment such as bacitracin or polymyxin B

Keratoconjunctivitis requires more frequent (as often as 2 hours, maximum 9 drops a day) trifluridine application

Cidofovir might be effective 
System, Problem, and Drug Regimen

Duration

Adverse Effects/Drug Interactions

Comments

\section{SKIN/MUCOCUTANEOUS (cont.)} Bacillary angiomatosis

Erythromycin $500 \mathrm{mg} 2$ months po qid

OR

Doxycycline $100 \mathrm{mg} \quad 2$ months po bid

\section{Eosinophilic folliculitis}

High-potency fluorinated Indefinitely corticosteroid cream bid

plus

Antihistamine (eg, diphen-Indefinitely

hydramine [Benadryl],

hydroxyzine [Atarax,

Vistaril], doxepin [Sinequan])

\section{HEMATOLOGIC}

Thrombocytopenia

Observation

\section{OPHTHALMOLOGIC \\ Cytomegalovirus (CMV) \\ Propbylaxis}

Ganciclovir (Cytovene) Indefinitely

$1 \mathrm{~g}$ po tid

See below

Acute retinitis

Induction

Ganciclovir $5 \mathrm{mg} / \mathrm{kg} /$ dose 14 days for IV a $12 \mathrm{~h}$; dosage acute retinal reduction in renal failure infection: 14 21 days usually required for extraocular infection

OR

Foscarnet (Foscavir) 14-day $90 \mathrm{mg} / \mathrm{kg} /$ dose $\mathrm{IV} \mathrm{q} 12 \mathrm{~h}$ induction as 2-hour infusion, dosage discontinuation or reduction in renal failure

OR

Ganciclovir plus foscarnet
See GENERAL/SYSTEMIC, MAC, clarithromycin, azithromycin. JarischHerxheimer reaction with systemic disease

Discontinue drugs that can cause thrombocytopenia

Corticosteroids can increase immunodeficiency

Neutropenia, leukopenia, anemia, thrombocytopenia (avoid if platelet count $<20,000 / \mu \mathrm{L}$ ); aminotransferase elevations; renal failure; phlebitis, rash; nausea. Discontinue zidovudine during induction to minimize additive hematologic toxicity (neutropenia). To avoid hematologic toxicity, substitute didanosine, zalcitabine, or stavudine for zidovudine, or change to foscarnet

Nephrotoxicity common; tremors, headaches, occasional seizures, muscle spasms; hypocalcemia, hypercalcemia, hypokalemia, hypophosphatemia, hyperphosphatemia; anemia, granulocytopenia; aminotransferase elevations; phlebitis, penile ulcerations

Drug interactions

Avoid concurrent use of nephrotoxic agents when possible

See individual agents above
Skin lesions can resolve in $1-3$ weeks, but 2 months' treatment needed. Systemic disease (eg, hepatic, splenic, central nervous system, bone) or cutaneous recurrences require treatment for 4 months or indefinitely. Azithromycin $1 \mathrm{~g}$ po qd and clarithromycin $500-1000$ mg po qd can be used as alternatives

Itraconazole $200 \mathrm{mg}$ po once daily with food might be effective. If no response in 2 weeks, increase dosage to $200 \mathrm{mg}$ po bid for 2 additional weeks. If no response after 4 weeks, discontinue. Topical metronidazole might be helpful

Avoid astemizole in combination with azole antibiotics because of increased risk of torsades de pointes and ventricular tachyarrhythmias

Treatment not required in absence of bleeding. Consider platelet transfusions prior to invasive procedures. High-dosage zidonudine, corticosteroids (eg, prednisone $60 \mathrm{mg}$ po $\mathrm{qd}$ ), splenectomy, intravenous gamma globulin, and interferon-alpha can raise platelet count

Oral ganciclovir primary prophylaxis is not currently recommended. Efficacy not established

Intravitreal ganciclovir by injection or implant appears effective if $I V$ causes unacceptable toxicity. Does not provide systemic therapeutic effect or protection of contralateral eye

Start G-CSF (Filgrastim, Neupogen) 300 $\mu \mathrm{g} S Q$ qd to 3 times a week for ganciclovirinduced neutropenia $(\mathrm{ANC}<500 / \mu \mathrm{L})$ on two consecutive measurements

Administered by infusion pump via central line. Infusion of $500-1000 \mathrm{~mL}$ normal saline before each foscarnet administration can minimize nephrotoxicity. Creatinine clearance should be measured in cachectic patients and in patients with renal insufficiency to ensure proper use of administration nomogram

Continue maintenance drug, induce with the alternative drug, then continue maintenance therapy with both drugs

Continued 
System, Problem, and Drug Regimen

\section{OPHTHALMOLOGIC}

Cytomegalovirus (CMV) (cont.)

Alternatives to ganciclovir or foscarnet

Cidofovir (Vistide) $5 \mathrm{mg}$ / $\mathrm{kg}$ IV with probenecid ( $\mathrm{g}$ po 3 hours before and $1 \mathrm{~g}$ po 2 and 8 hours after infusion) each week for 2 weeks, then every 2 weeks thereafter; contraindicated in renal insufficiency (serum creatinine $\geq 1.51$ $\mathrm{mg} / \mathrm{dL}, \mathrm{CrCl} \leq 55 \mathrm{~mL}$ min, $2+$ proteinuria)

\section{OR}

Ganciclovir implant (Vitasert)

$$
\text { plus }
$$

Ganciclovir 1g po tid

\section{Maintenance}

Ganciclovir $5 \mathrm{mg} / \mathrm{kg} \mathrm{IV}$ gd as 1-hour infusion; dosage reduction in renal failure

\section{OR}

Foscarnet $90 \mathrm{mg} / \mathrm{kg} \mathrm{IV}$ gd as 2-hour infusion; discontinuation or dosage reduction in renal failure

OR

Ganciclovir plus Indefinitely
foscarnet

14-day induction period

Indefinitely

Indefinitely

Indefinitely foscarnet

OR

Ganciclovir $1 \mathrm{~g}$ po tid
OR
Cidofovir $5 \mathrm{mg} / \mathrm{kg}$ as
1 -hour infusion every
2 weeks at infusion center
ORAL CAVTTY
Candida albicans
Clotrimazole (Mycelex)
troches $10 \mathrm{mg} 5$ times a
day or vaginal supposi-
tories $100 \mathrm{mg}$ qd - bid.
Dissolve troche slowly
in mouth

Indefinitely See above

Indefinitely

1 - 2 weeks or until resolved; maintenance (with lowest effective dosage) might be required for severe or frequent recurrences elevations
Life-threatening nephrotoxicity; iritis, ocular hypotonia; proteinuria, Indwelling catheter not required metabolic acidosis; neutropenia. Persons allergic to sulfa compounds Prehydrate with $1 \mathrm{~L}$ normal saline. can be allergic to probenecid

\section{Drug interactions}

Avoid concomitant administration with any potentially nephrotoxic agent, including nonsteroidal anti-inflammatory drugs

Implant can cause transient visual deterioration, retinal detachment, vitreal hemorrhage, cataracts, and endophthalmitis

Oral ganciclovir: Anemia, neutropenia; nephrotoxicity; neuropathy

\section{Drug interactions}

Oral ganciclovir therapy causes $50 \%$ increase in didanosine blood levels; reduce didanosine dosage by $50 \%$

See individual agents above cannot be given with potentially nephrotoxic drugs

Minimal toxicity. Unpleasant taste, nausea, vomiting; aminotransferase
Do not administer within 7 days of other potentially nephrotoxic agents. Patients previously treated with foscarnet are at increased risk for renal failure. Administer G-CSF if ANC consistently $<500 / \mu \mathrm{L}$

Implant ideal for patients unable to take daily intravenous therapy. Combine with oral ganciclovir to provide protection against systemic disease and contralateral eye involvement

Oral ganciclovir absorption is erratic when diarrhea is present. Administer on empty stomach to improve absorption

Lifelong suppressive therapy required to prevent recurrence of retinitis. Less frequent ( 5 - 6 days per week) administration might be acceptable. Administer G-CSF or change to foscarnet if ANC consistently < 500/ $\mathrm{\mu L}$

Maintenance with $120 \mathrm{mg} / \mathrm{kg} / \mathrm{d}$ might be more effective but also more toxic

Combination therapy not routinely recommended as initial therapy. Continue maintenance dosage of current drug; induce alternate drug, followed by maintenance with both drugs. Reinduction with ganciclovir or foscarnet might be helpful for recurrences when alternative drug cannot be administered

Oral ganciclovir is not as effective for maintenance therapy as other regimens

Does not require indwelling catheter; quality of life might be improved

Troches have high sugar content and often require frequent administration. Suppositories can be more convenient 
System, Problem, and

Duration

Adverse Effects/Drug Interactions

Comments

\section{ORAL CAVITY \\ Candida albicans (cont.)}

OR

Nystatin (Mycostatin) $100,000 \mathrm{U} / \mathrm{mL}$, swish and swallow $5 \mathrm{~mL}$ po q 6 h or one $500,000-\mathrm{U}$ tablet dissolved slowly in mouth $\mathrm{q} 6 \mathrm{~h}$

OR

Fluconazole (Diflucan) $100-200 \mathrm{mg}$ po qd therapy 50 - $100 \mathrm{mg}$ po qd; $100-200 \mathrm{mg}$ po once weekly is less effective

OR

Ketoconazole (Nizoral) $400 \mathrm{mg}$ po qd followed by maintenance therapy $200 \mathrm{mg}$ po qd - bid for 7 consecutive days per month

OR Amphotericin B oral
suspension $100 \mathrm{mg} / \mathrm{mL}$, swish and swallow

$1-5 \mathrm{~mL}$ qid

\section{Periodontal disease}

Hydrogen peroxide gargles for $30 \mathrm{sec}$ bid followed by maintenance

Same

Same

\section{See CENTRAL NERVO \\ Cryptococcus neoformans}

Large oral doses can produce diarrhea, nausea, vomiting

Nausea, vomiting; aminotransferase elevations; anaphylaxis, urticaria.

Higher dosages can suppress testosterone levels; gynecomastia; adrenal suppression

\section{Drug interactions}

Need gastric acidity to be effective; avoid antacids, $\mathrm{H}_{2}$ antagonists; administer 2 hours apart from didanosine. Higher dosages might be necessary if taking rifampin. Avoid concurrent use with triazolam or alprazolam

Same

Unpalatable; nausea, vomiting, diarrhea; rare urticaria

Not absorbed. No systemic effects. Intravenous amphotericin B might be necessary for severe disease

Indefinitely

OR

Chlorhexidine gluconate Indefinitely (Peridex) oral rinse $15 \mathrm{~mL}$ swished in mouth for $30 \mathrm{sec}$ bid

\section{ESOPHAGEAL.}

\section{Candida albicans}

Fluconazole $200-400 \mathrm{mg}$ po gd; higher dosages might be required

$14-21$ days; maintenance with lowest effective dosage
Generally less effective than ketoconazole, fluconazole, and clotrimazole. Can be effective in fluconazole-resistant candidal infection

Effective in oral candidiasis unresponsive to above oral agents. Higher dosages might be required. Fluconazole solution or itraconazole $200 \mathrm{mg}$ po qd (or intraconazole solution) might be effective against fluconazole-resistant Candida albicans removal of plaque are essential. Severe periodontal disease can require antibiotic therapy with metronidazole $250 \mathrm{mg}$ po tid or 7 - 10 days (alternatives: clindamycin or amoxicillin-clavulanate [Augmentin]). Antiseptic mouthwash (Listerine) gargles can be effective

Staining of teeth

See CENTRAL NERVOUS SYSTEM, Empiric treatment for patients with dysphagia or odynophagia who have oral thrush. Endoscopy with biopsy and cultures appropriate for patients who fail to respond within 1 week. Ketoconazole less expensive than fluconazole and effective in most patients
Oral hygiene measures with manual

\section{OR}

Ketoconazole $200 \mathrm{mg}$ po bid; see ORAL CAVIIY, Same as above See ORAL CAITTY, Candida albicans 
System, Problem, and

Drug Regimen

Duration

Adverse Effects/Drug Interactions

Comments

\section{ESOPHAGEAL}

Candida albicans (cont.)

OR

Amphotericin B

$0.3-0.4 \mathrm{mg} / \mathrm{kg} \mathrm{IV} \mathrm{qd}$

Cytomegalovirus

Ganciclovir; foscarnet see OPHTHALMO-

LOGIC, CMV

\section{Herpes simplex}

Acyclovir IV or valacyclovir po; see SKIN/ MUCOCUTANEOUS, disseminated, extensive, or persistent herpes simplex

\section{GASTROINTESTINAL \\ Nausea and vomiting \\ Prochlorperazine \\ (Compazine) 2.5 - 10.0 \\ $\mathrm{mg} \mathrm{IV}$ or $5-10 \mathrm{mg} \mathrm{po}$ \\ or IM q $6 \mathrm{~h}$, or $25 \mathrm{mg}$ po $\mathrm{q} 12 \mathrm{~h}$}

Metoclopramide

(Reglan) $10 \mathrm{mg}$ po qid, or $1 \mathrm{mg} / \mathrm{kg} \mathrm{Nq} 3 \mathrm{~h}$, or $10 \mathrm{mg}$ LMq $4-6 \mathrm{~h}$. Dosage reduction in renal failure

Lorazepam (Ativan) 0.5 $2.0 \mathrm{mg}$ po or SL tid - qid

Granisetron (Kytril) $1 \mathrm{mg}$ po $12 \mathrm{~h}$, or $10 \mu \mathrm{g} / \mathrm{kg}$ bid $I V$, or ondansetron (Zofran) $0.15 \mathrm{mg} / \mathrm{kg} \mathrm{IV}$ infusion for $15 \mathrm{~min} \mathrm{q} 6 \mathrm{~h}$ or $4-10 \mathrm{mg}$ po $\mathrm{q} 6 \mathrm{~h}$

Dronabinol (Marinol) 2.5 - $10.0 \mathrm{mg}$ po q 8 - $12 \mathrm{~h}$

Droperidol (Inapsine) $2.5 \mathrm{mg} \mathrm{LM} / \mathrm{IVq} 4-6 \mathrm{~h}$

\section{Diarthea}

Symptomatic treatment Loperamide (Imodium) $4 \mathrm{mg}$ po initially then $2 \mathrm{mg}$ $q 6 \mathrm{~h}$ around the clock and prn (maximum $16 \mathrm{mg} \mathrm{qd}$ )

Diphenoxylate-atropine (Lomotil) 2.5 - $5.0 \mathrm{mg}$ po 3 - 6 times daily for $24-48$ hours; then $2.5-5.0 \mathrm{mg}$ tid and prn to control diarrhea (maximum $20 \mathrm{mg} \mathrm{qd}$ )

Paregoric $0.4 \mathrm{mg}$ morphine/mL, $5-10 \mathrm{~mL}$ qd - qid, or tincture of opium $10 \mathrm{mg}$ morphine $/ \mathrm{mL}$. $0.3-1.0 \mathrm{~mL}$ po qid and $\mathrm{pm}$ (maximum $1 \mathrm{~mL} /$ dose or $6 \mathrm{~mL} / \mathrm{d}$ ), or equivalent
10 days or until resolution

\section{4 - 21 days See OPHTHALMOLOGIC, CMV}

10 - 14 days; maintenance required

As needed

Fatigue, drowsiness, dizziness, depression; extrapyramidal reactions; dystonic reactions; aminotransferase elevations; constipation

As needed

Same as above

As needed

As needed Similar

Constipation, diarrhea, abdominal pain; fever, chills; headache; sedation

As needed

As needed

As needed

Abdominal cramps, nausea abdominal distention, vomiting; dizziness, drowsiness

As needed

Ileus; nausea, vomiting, abdominal discomfort; anticholinergic side effects secondary to atropine

As needed

Ileus. Altered mental status hallucinations. Adverse effects common to narcotic analgesics
Candidal esophagitis unresponsive to oral agents requires low-dose $\mathrm{IV}$ amphotericin B

Diagnose by endoscopic appearance plus biopsy showing CMV inclusion bodies and positive culture. Long-term suppressive therapy indicated only after multiple recurrences. Beware of drug resistance

Diagnose by endoscopic appearance plus positive culture

Combinations of these agents often necessary

Haloperidol (Haldol) can also be effective

Same as above

Effective for anticipatory nausea

Reserved for intractable nausea and vomiting unresponsive to other agents. Ondansetron or granisetron in combination with droperidol helpful for intractable nausea and vomiting

Effective in drug-induced nausea. Marijuana can be helpful

Around-the-clock regimen more effective than prn. Treat to $2-3$ bowel movements per day

Same as above. $2.5 \mathrm{mg}$ diphenoxylateatropine is equivalent to $2 \mathrm{mg}$ morphine sulfate

Same as above. $5 \mathrm{~mL}$ paregoric and $0.2 \mathrm{~mL}$ tincture of opium are equivalent to $2 \mathrm{mg}$ morphine sulfate 
System, Problem, and

\section{GASTROINTESTINAL}

\section{Diarrhea (cont.)}

$14-21$ days

Indefinitely

21 days

See PULMONARY, PCP

\section{See OPHTHALMOLOGIC, CMV}

See TMP-SMX below drug resistance
Octreotide (Sandostatin) Indefinitely $100 \mu \mathrm{g}$ SQ tid, increase by $100-200 \mu g \mathrm{q} 1-2$

wk until maximum of

$500 \mu \mathrm{g} \mathrm{SQ}$ tid

\section{Cryptosporidium}

Paromomycin (Humatin)

$750 \mathrm{mg}$ po tid

$10-14$ days or indefinitely

\section{Isospora belli}

Trimethoprim-

sulfamethoxazole

(TMP-SMX, Septra,

Bactrim) 1 DS (double-

strength) tablet po qid

Cytomegalovirus

Ganciclovir; foscarnet; see OPHTHALMO-

LOGIC, CMV

\section{PULMONARY \\ Pneumocystis carinii pneumonia (PCP)}

Propbylaxis for patients with AIDS (including CD4+cell

count $<200 / \mu L)$, unexplained

fever, or oral candidiasis

Trimethoprim-

sulfamethoxazole (TMP-

SMX) 1 DS tablet po qd

or qod or 3 times a week

(eg, M-W-F)

Nausea, steatorrhea; hyperglycemia; pain at injection site

Nausea, vomiting, diarthea; rare ototoxicity and nephrotoxicity (similar to other aminoglycosides) only if absorbed through ulcerative bowel lesions

See dapsone plus TMP. Patients allergic to sulfa might tolerate dapsone; some cross-sensitivity

Headaches; nausea, diarrhea, aminotransferase elevations; rash, drug fever; neutropenia, anemia; transient conjunctivitis; erythema multiforme

Indefinitely

Inhaled pentamidine (Aeropent) $300 \mathrm{mg}$ $\mathrm{q} 4$ wk using Respirgard II nebulizer

\section{OR}

Clindamycin (Cleocin) Indefinitely 450 - $600 \mathrm{mg}$ po

bid - tid plus primaquine $15 \mathrm{mg}$ po $\mathrm{qd}$

Bronchospasm and coughing are common; pretreatment with inhaled Increased risk of spontaneous pneumothorax. Minimal systemic rare nephrotoxicity

See Acute PCP below
Not approved by FDA. Efficacy not demonstrated. Long-term safety unknown. Octreotide does not improve malabsorption

No evidence of efficacy. Addition of azithromycin $600 \mathrm{mg}$ po qod might increase effectiveness

\section{Usually effective}

Long-term suppressive therapy indicated only after multiple recurrences. Beware of

TMP-SMX considered most effective for prophylaxis. TMP-SMX provides additional prophylaxis against toxoplasmosis and common bacterial infections

Probably less effective than TMP-SMX; might be less toxic. Check glucose- 6 phosphate dehydrogenase (G6PD) before starting dapsone. Lower dosages (eg, 100 mg po 2 times a week) might be effective

Take with food to increase drug absorption. Patients with enteropathy might not absorb a sufficient amount of atovaquone for adequate treatment. Better tolerated than dapsone; efficacy similar

Effective for prophylaxis against primary PCP when CD4+ cell count $>150 / \mu \mathrm{L}$. bronchodilator ( $\mathrm{eg}$, albuterol) can help. Does not prevent extrapulmonary disease. Upper lobe recurrences from poor drug distribution when inhaled in upright posieffects. Rare pancreatitis, hypoglycemia; tion. Do not use in patients with possible $M$ tuberculosis infection because of risk of $M$ tuberculosis spread by aerosolization.

Efficacy and proper dosages for PCP prophylaxis unknown

Continued 
System, Problem, and

Drug Regimen

Duration

Adverse Effects/Drug Interactions

Comments

PULMONARY Pneumocystis carinii pneumonia (PCP) (cont.)

OR

Pyrimethamine

$25 \mathrm{mg}$ - sulfadoxine $500 \mathrm{mg}$

(Fansidar) 1 po q 2 wk

TMP-SMX; TMP doses either po or as 1- to

2-hour IV infusions; lower dosages (TMP $12 \mathrm{mg} / \mathrm{kg} / \mathrm{d}$ )

can be effective and less toxic

Note: Patients with substantial hypoxemia require concomitant corticosteroids (see below)
Acute PCP

$15 / \mathrm{kg} / \mathrm{d}$ given in 3 divided

Indefinitely

Stevens-Johnson syndrome, toxic epidermal necrolysis; bone marrow suppression; gastrointestinal, central nervous system toxicity

Adverse effects commonly appear between 7 and 14 days in more than $50 \%$ of patients

Rashes: maculopapular, exfoliative, Stevens-Johnson syndrome

Hematologic: neutropenia, leukopenia, thrombocytopenia, anemia

Drug interactions

Concurrent leucovorin calcium therapy associated with increased rate of therapeutic failure

Gastrointestinal: nausea, vomiting, aminotransferase elevations

Renal: increased blood urea nitrogen (BUN) and creatinine; hyperkalemia secondary to effects of TMP

Hyponatremia

Neurologic: tremor, psychosis, aseptic meningitis

Drug fever. Sepsis-like syndrome, especially upon rechallenge

Alternatives to TMP-SMX for acute PCP Pentamidine isethionate 21 days (Pentam) $4 \mathrm{mg} / \mathrm{kg} / \mathrm{d}$ as 1- to 2-hour IV infusion once a day; $3 \mathrm{mg} / \mathrm{kg} / \mathrm{d}$ might also be effective
Adverse effects commonly appear between 7 and 14 days

Orthostatic hypotension can be severe and occur with initial infusion

Pancreatitis; early or delayed hypoglycemia (can occur after discontinuation of therapy); hyperglycemia

Drug interactions

Avoid concomitant pancreatic toxins such as didanosine, zalcitabine, and alcohol
No studies clearly show efficacy

TMP-SMX is the drug of choice and should be used unless severe reactions (eg, anaphylaxis, Stevens-Johnson syndrome) are of concern. Oral and intravenous routes equally effective

Mild rash does not necessitate stopping or changing treatment: institute antihistamine or rechallenge with lower dosage of TMP-SMX. Desensitization more successful than rechallenge.

If $\mathrm{ANC}<500 / \mu \mathrm{L}$ or if platelet count $<30 \times 10^{9} / \mathrm{L}$ and bleeding occurs, consider alternative treatment

Pretreatment with lorazepam, prochlorperazine, metoclopramide, or dronabinol to reduce nausea. Nausea can be less with oral TMP-SMX. Aminotransferase elevations 4- 5 times normal require treatment change

TMP decreases creatinine tubular secretion and can elevate serum creatinine levels. Discontinue TMP-SMX if serum creatinine $>3.0 \mathrm{mg} / \mathrm{dL}$

Can be caused by large volume of $5 \%$ dextrose in water (D $5 W$ ) needed for $I V$ administration; dilute each $80 \mathrm{mg}$ TMP in $75 \mathrm{~mL}$ DSW or change to oral TMPSMX. For severe hyponatremia $\left(\mathrm{Na}^{+}<115\right.$ $\mathrm{mEq} / \mathrm{dL}$ ), can dilute in normal saline; administer within 1 hour of preparation to avoid TMP-SMX precipitation

Tremors can be confused with seizures

Drug fever can herald onset of neutropenia, rash, hepatitis, and bone marrow toxicity

Slow IV infusion for 2 hours can prevent hypotension. Check blood pressure at end of infusion

Hypoglycemia can be profound and prolonged, requiring immediate IV D50W followed by D10W glucose infusions. Permanent diabetes can occur. 
System, Problem, and

$\begin{array}{lll}\text { Drug Regimen } & \text { Duration Adverse Effects/Drug Interactions } & \text { Comments }\end{array}$

PULMONARY Pneumocystis carinii pneumonia (PCP) (cont.)

Pentamidine isethionate (cont.)

OR

Clindamycin $600 \mathrm{mg}$ IV 21 days
or po tid

plus

Primaquine $30-\mathrm{mg}$ base po qd

OR

Dapsone $50 \mathrm{mg}$ po bid plus either TMP $15 \mathrm{mg} /$ $\mathrm{kg} / \mathrm{d}$ po in $3-4$ divided doses or pyrimethamine $50-75 \mathrm{mg}$ po gd

OR

Trimetrexate (Neutrexin) 21 days $45 \mathrm{mg} / \mathrm{m} 2 \mathrm{IV}$ qd

plus

Dapsone $50 \mathrm{mg}$ po bid

plus

Leucovorin calcium (folinic acid) $20 \mathrm{mg} / \mathrm{m} 2 \mathrm{IV}$ or po $q 6 \mathrm{~h}$

OR

Atovaquone suspension

$(750 \mathrm{mg} / 5 \mathrm{~mL}) 750 \mathrm{mg}$ po

21 days bid with food

plus

Pyrimethamine $50-75 \mathrm{mg}$ po qd

\section{1 days}

Renal failure; hyperkalemia. Concomitant nephrotoxic agents (eg, nonsteroidal antiinflammatory agents) and dehydration increase risk of nephrotoxicity

Rare: neutropenia, thrombocytopenia; hypocalcemia, hypomagnesemia; aminotransferase elevations; cardiac arrhythmias (rare) with prolongation of QT interval and $T$-wave flattening

Maculopapular rash (day 10 - 12 most common, usually self-limiting), fever; diarrhea, nausea, vomiting, abdominal cramps, Clostridium difficile colitis, aminotransferase elevations

Methemoglobinemia from primaquine, hemolysis in G6PD-deficient patients; leukopenia

See toxicities for TMP-SMX. Patients allergic to sulfa often tolerate dapsone. Methemoglobinemia, dose-related hemolysis, bone marrow suppression; rash; fever; nausea, abdominal pain; hyperkalemia; proteinuria, papillary necrosis

\section{Drug interactions}

Drug interactions with rifampin and rifabutin can render dapsone ineffective

Granulocytopenia, fever, rash; aminotransferase elevations

\section{1 days See above}

Rash, drug fever; headaches; nausea, diarrhea, aminotransferase elevations; neutropenia, anemia; transient conjunctivitis; erythema multiforme
Consider in patients with mild-tomoderate PCP, intolerant of or unresponsive to TMP-SMX

Check G6PD before initiating primaquine therapy. Check methemoglobin levels when clinically indicated (see dapsone). Vitamin $C 1$ g po tid might prevent methemoglobinemia. Lower dosage of primaquine ( $15 \mathrm{mg}$ po qd) can be effective

Effective in mild-to-moderate PCP only. Check G6PD before starting dapsone. Check methemoglobin levels if symptomatic or discrepancy between oxygen saturation and simultaneous arterial $\mathrm{PaO}_{2}$. Treat methemoglobinemia $>20 \%(13 \%$ $20 \%$ if anemic or respiratory compromise) with methylene blue $1 \%$ solution $2 \mathrm{mg} / \mathrm{kg}$ IV once; methylene blue contraindicated in G6PD deficiency. Vitamin C $1 \mathrm{~g}$ po tid might prevent methemoglobinemia

Can be effective in some patients as salvage therapy

Must be administered for 72 hours after the last dose of trimetrexate. Large IV fluid load with leucovorin administration can result in volume overload

Higher therapeutic failure rate than TMP-SMX. For patients who fail or are intolerant of TMP-SMX, pentamidine, dapsone-TMP, or clindamycin-primaquine. Take with food to increase drug absorption. Patients with enteropathy might not absorb a sufficient amount of atovaquone for adequate treatment

Corticosteroids indicated in conjunction with antipneumocystis therapy in all patients with $\mathrm{PaO}_{2} \leq 70 \mathrm{mmHg}$. Begin corticosteroids concurrently with PCP treatment or if $\mathrm{PaO}_{2}$ decreases to $\leq 70 \mathrm{mmHg}$ within 72 hours of initiating PCP treatment.

Continued 
System, Problem, and Drug Regimen

Duration

Adverse Effects/Drug Interactions

Comments

CENTRAL NERVOUS SYSTEM

Toxoplamma gondii

Prophylaxis

Most PCP prophylaxis

regimens provide some

Indefinitely See PULMONARY, PCP

protection against

toxoplasmosis

\section{Acute}

Pyrimethamine

$75-100 \mathrm{mg}$ po qd (every other day if bone marrow suppression) plus leucovorin calcium (folinic acid) $10-25 \mathrm{mg}$ po qd

plus either

Sulfadiazine $1.0-1.5 \mathrm{~g}$ po q $6 \mathrm{~h}$

or

Clindamycin 600 $900 \mathrm{mg}$ po or IV qid

Alternative when intolerant of sulfadiazine and clindamycin

Pyrimethamine plus

leucovorin as above

plus one of the following

Clarithromycin $1 \mathrm{~g}$ po

bid or azithromycin

$1200-1500 \mathrm{mg}$ po qd

or

Atovaquone suspension $(750 \mathrm{mg} / 5 \mathrm{~mL}) 750 \mathrm{mg}$ po qid with meals

or

Doxycycline $100 \mathrm{mg}$ po tid - qid or minocycline

$200 \mathrm{mg}$ po bid

or

Dapsone $100 \mathrm{mg}$ po qd

or

Pyrimethamine alone

$100-200 \mathrm{mg}$ po qd

OR

TMP/SMX as for acute PCP Same
6 - 8 weeks

for acute

therapy

Same

Same

Same

Same

Same

Same

Same

Same

Rash, drug fever; leukopenia, thrombocytopenia; crystalluria with renal failure

See PULMONARY, PCP

See above

See GENERAI/SYSTEMIC, MAC

See PULMONARY, PCP

Tetracycline side effects

See PULMONARY, PCP

See PULMONARY, PCP

Not as effective as above regimens
Not proved effective

Not proved effective
TMP-SMX, dapsone plus TMP or pyrimethamine, clindamycin plus primaquine, atovaquone plus pyrimethamine, and pyrimethamine-sulfadoxine provide some prophylaxis against toxoplasmosis. Aerosolized pentamidine not effective; adding another agent to provide toxoplasmosis prophylaxis not required. Clarithromycin and azithromycin provide some benefit

Clinical response or regression of lesions on imaging studies is usually noted within 2 weeks. Maintenance required indefinitely to prevent relapse

Sulfadiazine probably provides effective prophylaxis against PCP. Ensure adequate fluid intake
Maintenance

Pyrimethamine

25 - $50 \mathrm{mg}$ po qd

Indefinitely

See PULMONARY, PCP

plus either

Sulfadiazine $1 \mathrm{~g}$ po $\mathrm{q} 12 \mathrm{~h} \quad$ Indefinitely

or

Clindamycin 300-450 mg Indefinitely po $\mathrm{q} 6-8 \mathrm{~h}$

Continued of leukopenia 


\section{CENTRAL NERVOUS}

\section{SYSTEM}

Cryptococcus neoformans

\section{Prophylaxis}

Fluconazole provides

limited prophylaxis

\section{Meningitis or disseminated cryptococcosis \\ Acute}

Amphotericin B 0.7 - 1.0 $\mathrm{mg} / \mathrm{kg} / \mathrm{d}$ IV with or without 5-flucytosine

(Ancobon) $100 \mathrm{mg} / \mathrm{kg}$ po

gd in 4 divided doses for

first $2-4$ weeks. If

clinically improved after

$7.5 \mathrm{mg} / \mathrm{kg}$ total amphotericin $\mathrm{B}$ administration, can change to fluconazole $400 \mathrm{mg}$ po $\mathrm{qd}$ or itraconazole $200 \mathrm{mg}$ po bid

\section{OR}

Fluconazole 400 - $800 \mathrm{mg}$ po qd. Dosage reduction in renal failure. Higher dosages (eg, 800 - $1200 \mathrm{mg}$ po qd) might increase efficacy

\section{Maintenance}

Fluconazole $200-400 \mathrm{mg}$ po qd

\section{OR}

Amphotericin B

$0.5-0.8 \mathrm{mg} / \mathrm{kg} / \mathrm{d}$

3 - 5 times a week

\section{Syphilis}

Aqueous crystalline penicillin G $3-4 \mathrm{mU}$ IV q $4 \mathrm{~h}$ (total $18-24 \mathrm{mU} / \mathrm{d}$ )

\section{OR}

Procaine penicillin $\mathrm{G}$ $2.4 \mathrm{mU} \mathrm{IM} \mathrm{qd}$

$$
\text { plus }
$$

Probenecid $500 \mathrm{mg}$ po qid
$8-12$

weeks

$10-14$ days

Usual penicillin adverse effects, Jarisch-Herxheimer reaction; seizures from high-dosage penicillin in renal failure

10-14 days Same. Probenecid rash aminotransferase elevations; rare alopecia

\section{Drug interactions}

Increased phenytoin (Dilantin) and warfarin (Coumadin) levels; higher for patients taking rifampin

\section{Indefinitely Same}

Indefinitely Same
Primary prophylaxis not routinely recommended. Can be considered for patients with CDH+ cell counts $<50 / \mu \mathrm{L}$. No long-term survival benefit.

Fluconazole resistance reported

6 - 8 weeks; Renal failure, hypokalemia, hypomagampho- nesemia. Liposomal amphotericin B tericin total might decrease toxicity

dosage exceed $2 \mathrm{~g}$

Fever, chills; anemia, thrombophlebitis

Granulocytopenia; nausea, vomiting, diarrhea, aminotransferase elevations; rash from flucytosine

Flucytosine toxicities (rash, metallic taste, leukopenia, thrombocytopenia) limit its usefulness

Nausea, vomiting, diarrhea; dizziness; cutaneous reactions, skin pigmentation, fuconazole dosages might be necessary

•
Pretreatment with diphenhydramine, acetaminophen or IV morphine can decrease amphotericin-induced fevers, chills, and rigors. Pretreatment not recommended routinely. Administer for $4-6$ hours in D5W. Addition of heparin $500 \mathrm{U}$ and hydrocortisone $25 \mathrm{mg}$ to amphotericin IV solution can decrease phlebitis. Infusion of $500-1000 \mathrm{~mL}$ normal saline before administration of amphotericin B can minimize renal toxicity. 5-Flucytosine not indicated if granulocytopenia or thrombocytopenia is present

Markedly increased intracranial pressure (> $240 \mathrm{~mm}$ ) might require cerebrospinal fluid drainage $(20-30 \mathrm{~mL}$ or more per day by lumbar puncture or continuous lumbar drain), or possibly corticosteroid, mannitol, or acetazolamide (Diamox) therapy

As effective as amphotericin B against mild or moderate disease; unknown whether equally effective against severe disease. Fluconazole penetrates central nervous system and most body tissues, including prostate. Addition of 5-flucytosine might be of benefit Higher dosages might be necessary for
recurrent disease

Continued serologic and clinical follow-up required to assess adequacy of treatment for neurosyphilis. Persons with ophthalmic auditory, or cranial nerve abnormalities or other syndromes consistent with neurosyphilis should receive daily penicillin therapy for $10-14$ days. Intravenous penicillin preferred for adequate central nervous system penetration. For penicillin-allergic patients, consultation with an expert actised. Administer additional benzathine penicillin $2.4 \mathrm{mU}$ LM weekly after completion of neurosyphilis treatment to ensure 3 weeks total penicillin therapy 
Table 1. Continued

System, Problem, and Drug Regimen

Duration

Adverse Effects/Drug Interactions

Comments

\section{CENTRAL}

NERVOUS SYSTEM

Peripheral neuropathy

Desipramine (Norpramin) or amitriptyline (Elavil)

25 - $150 \mathrm{mg}$ po hs

Indefinitely

Usual tricyclic side effects; drowsiness; orthostatic hypotension; anticholinergic symptoms

Phenytoin (diphenylhydantoin, Dilantin) $100 \mathrm{mg}$ po tid

Carbamazepine (Tegretol) 100 - 300 mg po bid

Mexiletine (Mexitil)

$150 \mathrm{mg}$ po bid - tid

Capsaicin (Axsain,

Zostrix-HP) $0.075 \%$

topical cream qid

Gabapentin (Neurontin)

$300-400 \mathrm{mg}$ po tid via dose escalation; dosage reduction in renal failure
Indefinitely

Indefinitely

Usual side effects and drug-drug interactions

Leukopenia, bone marrow suppression, rare agranulocytosis; rash; drowsiness, dizziness; aminotransferase elevations

Indefinitely Nausea, vomiting, epigastric pain; dizziness, tremor; bradycardia; rare seizures, leukopenia, agranulocytosis

Indefinitely Minor burning sensation, skin irritation, erythema

Indefinitely Thrombocytopenia; somnolence, dizziness, ataxia, nystagmus, fatigue, somnolence, headache; nausea, vomiting, diarrhea
Pain relief occurs in 3 - 5 days. Desipramine causes less sedation and fewer anticholinergic effects. Other tricyclic drugs might be equally effective

Generally ineffective

Less desirable because of bone marrow effects. Need to monitor carbamazepine levels to avoid toxicity

Less desirable because of side effects

Pain relief delayed 2 - 4 weeks. No systemic effects

Can be helpful when other agents fail 
principally in order of efficacy. Where more than one drug is effective for a specific condition, recommendations are usually in an order that favors the least expensive choice among equally effective options. The most common and clinically important adverse effects and drug interactions are listed.

\section{References}

A selected bibliography highlights the most important management and therapeutic problems associated with HIV infection and AIDS. References including articles about $P$ carinii pneumonia ${ }^{21}$ and other mycobacterial diseases ${ }^{22}$; dermatologic, ${ }^{23-25}$ oropharyngeal, ${ }^{26,27}$ ophthalmologic, ${ }^{28-30}$ and gastrointestinal problems ${ }^{31,32}$; the AIDS wasting syndrome ${ }^{33-35}$; and neurologic disease. ${ }^{36,37}$ Additional references are intended to assist primary care clinicians ${ }^{38}$ with the broad spectrum of problems associated with HIV infection and AIDS, ${ }^{39,40}$ other sexually transmitted diseases, ${ }^{41}$ and special treatment considerations for occupational exposures ${ }^{42-45}$ and pregnancy. ${ }^{46}$

\section{Other Sources}

Information about clinical trials is available through the AIDS Clinical Trials Information Service at 1-800-TRIALS A, and through the AIDS Treatment Information Service (ATIS) at 1800-HIV-8440. ATIS also has printed and Website guidelines and information about approved therapies and management protocols at http:// hivatis.org. Our National HIV Telephone Consultation Service (Warmline) at the University of California, San Francisco, Department of Family and Community Medicine at San Francisco General Hospital provides clinical consultation and education for health care providers; the Warmline is in operation on weekdays at 1-800-933-3413. The AIDS Education and Training Centers (AIDS ETCs) of the Health Resources and Services Administration (HRSA) at 1-301-443-6364 offers education, training, and consultation services to health care providers. HRSA also offers special-topic teleconferences. Additional information can be accessed at other Websites, including the San Francisco General Hospital-based site at http://www.hivinsite.ucsf.edu and the American Medial Association HIV/AIDS site at http://www. ama-assn.org.
We gratefully acknowledge the staff of the HIV Telephone Consultation Service and the faculty, staff, and house staff at San Francisco General Hospital for making this work possible, and Mary A. Hanville for assistance in preparation of this manuscript.

\section{References}

1. Goldschmidt RH, Dong BJ. Treatment of AIDS and HIV-related conditions - 1997. J Am Board Fam Pract 1997:10:144-67.

2. Report of the NIH Panel to Define Principles of Therapy of HIV Infection and guidelines for the use of antiretroviral agents in HIV-infected adults and adolescents. MMWR Morb Mortal Wkly Rep 1998; 47(RR-5):1-82. Also available at http://www.hivatis. org.

3. Carpenter CC, Fischl MA, Hammer SM, Hirsch MS, Jacobsen DM, Katzenstein DA, et al. Antiretroviral therapy for HIV infection in 1998. Updated recommendations of the International AIDS Society - USA Panel. JAMA 1998;280:78-86.

4. Gazzard B, Moyle G, on behalf of the BHIIVA Guidelines Writing Committee. 1998 revision to the British HIV Association guidelines for antiretroviral treatment of HIV seropositive individuals. Lancet 1998;352:314-6.

5. Goldschmidt RH, Balano KB, Legg JJ, Dong BJ. Individualized strategies in the era of combination antiretroviral therapy. J Am Board Fam Pract 1998;11: 158-64.

6. Maenza J, Flexner C. Combination antiretroviral therapy for HIV infection. Am Fam Physician 1998; 57:2789-98.

7. Volberding PA, Deeks SG. Antiretroviral therapy for HIV infection: promises and problems. JAMA 1998;279:1343-4.

8. Levy JA. Caution: should we be treating HIV infection early? Lancet 1998;352:982-3.

9. Flexner C. HIV-protease inhibitors. N Engl J Med 1998;338:1281-92.

10. Lisziewicz J, Jessen H, Finzi D, Siliciano RF, Lori F. HIV-1 suppression by early treatment with hydroxyurea, didanosine, and a protease inhibitor. Lancet 1998;352:199-200.

11. Hirsch MS, Klibanski A. Editorial response: What price progress? Pseudo-Cushing's syndrome associated with antiretroviral therapy in patients with human immunodeficiency virus infection. Clin Infect Dis 1998;27:73-5.

12. Lo JC, Mulligan K, Tai VW, Algren H, Schambelan M. "Buffalo hump" in men with HIV-1 infection. Lancet 1998;351:867-70.

13. Carr A, Samaras K, Chisholm KF, Cooper DA. Pathogenesis of HIV-1-protease inhibitor-associated peripheral lipodystrophy, hyperlipidaemia, and insulin resistance. Lancet 1998;352:1881-3.

14. Burman WJ, Reves RR, Cohn DL. The case for conservative management of early HIV disease. JAMA 1998;280:93-5. 
15. Palella FJ Jr, Delaney KM, Moorman AC, Loveless MO, Fuhrer J, Satten GA, et al. Declining morbidity and mortality among patients with advanced human immunodeficiency virus infection. HIV Outpatient Study Investigators. N Engl J Med 1998;338:853-60.

16. Sepkowitz KA. Effect of HAART on natural history of AIDS-related opportunistic disorders. Lancet 1998; 351:228-30.

17. 1997 USPHS/IDSA guidelines for the prevention of opportunistic infections in persons infected with human immunodeficiency virus. MMWR Morb Mortal Wkly Rep 1997;46(RR-12): 1-46.

18. Tural C, Romeu J, Sirera G, Andreu D, Conejero M, Ruiz S, et al. Long-lasting remission of cytomegalovirus retinitis without maintenance therapy in human immunodeficiency virus-infected patients. J Infect Dis 1998;177:1080-3.

19. Macdonald JC, Torriani FJ, Morse LS, Karavellas MP, Reed JB, Freeman WR. Lack of reactivation of cytomegalovirus (CMV) retinitis after stopping CMV maintenance therapy in AIDS patients with sustained elevations in CD4 $\mathrm{T}$ cells in response to highly active antiretroviral therapy. J Infect Dis 1998;177:1182-7.

20. Centers for Disease Control and Prevention. Prevention and treatment of tuberculosis in patients infected with human immunodeficiency virus. Principles of therapy and revised recommendations. MMWR Morb Mortal Wkly Rep 1998; 47(RR-20):1-58. Available at http://www.cdc.gov.

21. Masur H. Prevention and treatment of pneumocystis pneumonia. N Engl J Med 1992;327:1853-60.

22. Horsburgh CR Jr. Advances in the prevention and treatment of Mycobacterium avium disease. $\mathrm{N}$ Engl J Med 1996;335:428-30.

23. Tschachler E, Bergstresser PR, Stingl G. HIV-related skin diseases. Lancet 1996;348:659-63.

24. Cohen PR, Grossman ME. Recognizing skin lesions of systemic fungal infections in patients with AIDS. Am Fam Physician 1994;49:1627-34.

25. Coopman SA, Johnson RA, Platt R, Stern RS. Cutaneous disease and drug reactions in HTV infection. $N$ Engl J Med 1993;328:1670-4.

26. Greenspan D, Greenspan JS. HIV-related oral disease. Lancet 1996;348:729-33.

27. Weinert $M$, Grimes RM, Lynch DP. Oral manifestations of HIV infection. Ann Intern Med 1996;125: 485-96.

28. Cunningham ET Jr, Margolis TP. Ocular manifestations of HIV infection. N Engl J Med 1998;339:236-44.

29. Jacobson MA. Treatment of cytomegalovirus retinitis in patients with the acquired immunodeficiency syndrome. N Engl J Med 1997;337:105-14.

30. Whitley RJ, Jacobson MA, Friedberg DN, Holland GN, Jabs DA, Dieterich DT, et al. Guidelines for the treatment of cytomegalovirus diseases in patients with AIDS in the era of potent antiretroviral therapy: rec- ommendations of an international panel. Arch Intern Med 1998;158:957-69.

31. Sharpstone D, Gazzard B. Gastrointestinal manifestations of HIV infection. Lancet 1996;348:379-83.

32. DuPont HL, Marshall GD. HIV-associated diarrhoea and wasting. Lancet 1995;346:352-6.

33. Macallan DC, Noble C, Baldwin C, Jebb SA, Prentice AM, Coward WA, et al. Energy expenditure and wasting in human immunodeficiency virus infection. N Engl J Med 1995;333:83-8.

34. Grinspoon S, Corcoran C, Askari H, Schoenfeld D, Wolf L, Burrows B, et al. Effects of androgen administration in men with the AIDS wasting syndrome. A randomized, double-blind, placebo-controlled trial. Ann Intern Med 1998;129:18-26.

35. Cofrancesco J Jr, Whalen JJ 3rd, Dobs AS. Testosterone replacement treatment options for HIV-infected men. J Acquir Immune Defic Syndr Hum Retrovirol 1997;16:254-65.

36. Simpson DM, Tagliati $M$. Neurologic manifestations of HIV infection. Ann Intern Med 1994;121:769-85.

37. Newton HB. Common neurologic complications of HIV-1 infection and AIDS. Am Fam Physician 1995;51:387-98.

38. Soloway B. A new era in HIV care. Am Fam Physician 1997;56:37, 40-2.

39. Legg JJ, Balano KB. Symptom management in HIVinfected patients. Prim Care 1997;24:597-606.

40. Fournier AM, Carmichael C. Socioeconomic influences on the transmission of human immunodeficiency virus infection: the hidden risk. Arch Fam Med 1998;7:214-7.

41. 1998 guidelines for treatment of sexually transmitted diseases. Centers for Disease Control and Prevention. MMWR Morb Mortal Wkly Rep 1998;47(RR-1):1111.

42. Public Health Service guidelines for the management of health-care worker exposures to HIV and recommendations for postexposure prophylaxis. Centers for Disease Control and Prevention. MMWR Morb Mortal Wkly Rep 1998;47(RR-7):1-33.

43. Gerberding JL. Prophylaxis for occupational exposure to HIV. Ann Intern Med 1996;125:497-501.

44. Gerberding JL. Management of occupational exposures to blood-borne viruses. N Engl J Med 1995;332: 444-51.

45. Goldschmidt RH, Legg JJ, Balano KB. Occupational exposure to HIV: new recommendations for treating health care workers. J Am Board Fam Pract 1996;9: 455-8.

46. Public Health Service Task Force recommendations for the use of antiretroviral drugs in pregnant women infected with HIV-1 for maternal health and for reducing perinatal HIV-1 transmission in the United States. Centers for Disease Control and Prevention. MMWR Morb Mortal Wkly Rep 1998;47(RR-2):1-30. 